\title{
Modified Murray Loop Method for Underground Cable Fault Location Detection Using GSM
}

\author{
Snehal C. Kor ${ }^{1}$, Vivek D. Bavdhane ${ }^{2}$ \\ ${ }^{1,2}$ Zeal College of Engineering and Research, Narhe, Pune, Maharashtra, India
}

\begin{abstract}
In recent years, power systems have been very difficult to manage as the load demands increase and environment constraints restrict the distribution network. One another mode used for distribution of Electrical power is making use of underground cables (generally in urban areas only) instead of overhead distribution network. The use of underground cables arise a problem of identifying the fault location as it is not open to view as in case of overhead network. To improve the reliability of a distribution system, accurate identification of a faulted segment is required in order to reduce the interruption time during fault. Speedy and precise fault location plays an important role in accelerating system restoration, reducing outage time, reducing great financial loss and significantly improving system reliability. The objective of this paper is to study the methods of determining the distance of underground cable fault from the base station in kilometers. Underground cable system is a common practice followed in major urban areas. While a fault occurs for some reason, at that time the repairing process related to that particular cable is difficult due to exact unknown location of the fault in the cable. In this paper, a technique for detecting faults in underground distribution system is presented. Proposed system is used to find out the exact location of the fault and to send an SMS with details to a remote mobile phone using GSM module.
\end{abstract}

Keywords: Reliability, distribution system, underground cables, Murray loop test

\section{Introduction}

The main function of the electrical transmission and distribution systems is to transport electrical energy from the generation unit to the customers. Generally, when fault occurs on transmission lines, detecting fault is necessary for power system in order to clear fault before it increases the damage to the power system. Although the underground cable system provides higher reliability than the overhead line system, it is hard to seek out the fault location. The demand for reliable service has led to the development of technique of locating faults. During the course of recent years, the development of the fault diagnosis has been progressed with the applications of signal processing techniques and results in transient based techniques. It has been found that the wavelet transform is capable of investigating the transient signals generated in power system. The location of fault using wavelet transform was initially proposed by F. H. Magnago et al. Recently, several techniques have been employed to determine the fault location in underground cable such as age cable, bridge technique, Murray loop pulse radar and traveling wave but each technique has different solutions. In addition, a technique selection is available for fault locating; it depends on several factors such as length of circuit (or cable) and type of fault (sustained or temporary), etc.

\section{Murray Loop Test}

Murray loop test, Varley loop test and Pulse Echo test are simple basic method to localize cable fault testing. This method used basic equipment that obtained easily. These tests are performed for the location of either an earth fault or short circuit fault in underground cable. In these tests the resistance of fault does not affect the results obtained except when the resistance of fault is very high. There are two loop tests usually used and are known as MurrayLoop and Varley Loop Test. These tests imply the principle of Wheatstone bridge.
This test is used to find the fault location in an underground cable by making one Wheatstone bridge in it and by comparing the resistance we shall find out the fault location. But we should use the known length of the cables in this experiment. The necessary connection of the Murray loop test is shown in figure 2 and 3 . The figure 2 shows that the circuit connection for finding the fault location when the ground fault occurs and the figure 3 shows that the circuit connections for finding the fault location when the short circuit fault occurs.

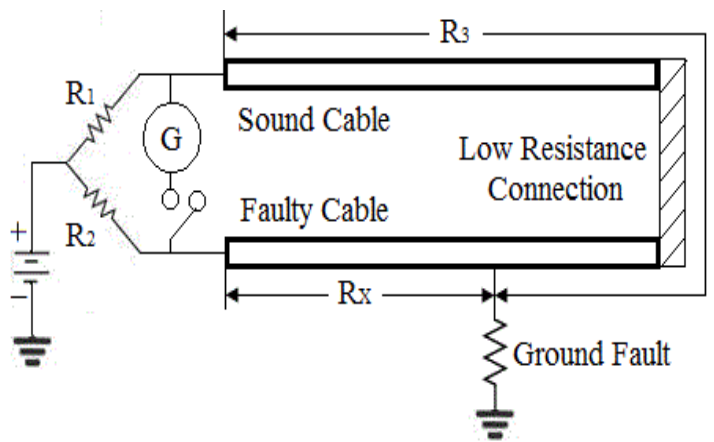

Figure 1: Circuit Connection of Murray Loop test for ground fault.

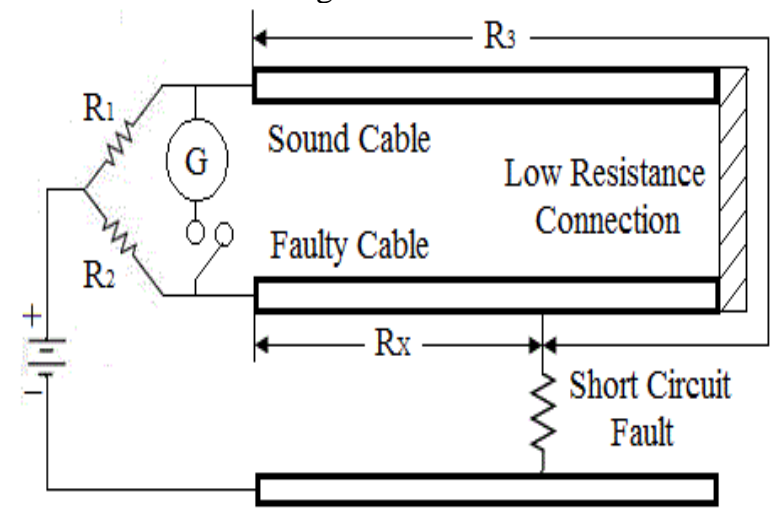

Figure 2: Circuit Connection of Murray Loop test for short Circuit fault. 


\section{International Journal of Science and Research (IJSR) \\ ISSN (Online): 2319-7064}

Index Copernicus Value (2013): 6.14 | Impact Factor (2015): 6.391

In this test, the faulty cable is connected with sound cable by a low resistance wire, because that resistance should not affect the total resistance of the cable and it should be able to circulate the loop current to the bridge circuits without loss.

The variable resistors $R_{1}$ and $R_{2}$ are forming the ratio arms. Balance of the bridge is achieved by adjusting the variable resistors. ' $G$ ' is the galvanometer to indicate the balance. $\left[\mathrm{R}_{3}\right.$ $\left.+R_{X}\right]$ is the total loop resistance formed by the sound cable and the faulty cable. At the balance condition,

$$
\begin{aligned}
& \frac{R_{1}}{R_{2}}=\frac{R_{3}}{R_{X}} \Rightarrow \frac{R_{1}+R_{2}}{R_{2}}=\frac{R_{3}+R_{X}}{R_{X}} \\
& \Rightarrow R_{X}=\frac{R_{2}}{R_{1}+R_{2}}\left(R_{3}+R_{X}\right)
\end{aligned}
$$

When the cross section area of the both sound cable and faulty cable are equal, then the resistance of the conductors are directly proportional to their lengths. So, if $\mathrm{L}_{X}$ represents the length between test end to the fault end of the faulty cable and if L represents the total length of the both cables, then the expression for $\mathrm{L}_{\mathrm{X}}$ is as follows;

$$
L_{X}=\frac{R_{2}}{R_{1}+R_{2}} L
$$

The above test is only valid when the lengths of the cables are known. In Murray Loop Test, the fault resistance is fixed and it may not be varied. Also it is difficult to set the bridge as balance. Thus, the determination of the fault position is not accurate. Then the current circulation through the cable would cause temperature rises due to high voltage or high current. If the resistance varies according to the temperature, then the balance collapses. So, we need to apply less voltage or less current to this circuit.

\section{Types of Fault Detection}

The faults occurring in the power lines and cables can be classified into four main categories - short circuit to another conductor in the cable, short circuit to earth, high resistance to earth and open circuit. Not all approaches work best for each type of fault. Four methods that are mostly used in detecting fault location are described as follows.

- A-Frame

- Thumper

- Time Domain Reflectometer (TDR)

- Bridge methods

\section{Requirement of Preliminary Investigations}

There are different types of high voltage cables as per their insulation, voltage resistivity, and potential separation of conductors from each other and from the metallic outer lead sheath or armor. Some of the examples are Mass Impregnated Paper (PILC), Polyvinylchloride (PVC), Cross Linked Polyethylene (XLPE), Ethylene Propylene Rubber (EPR), paper with oil and gas. While there are some other cables with various types of insulation materials, the above mentioned cables are most commonly used.

When a dc high voltage pulse is sent through any of these cables mentioned above, it incurs some capacitive charging current, insulation absorption current, insulation leakage current, and current at the fault to some degree. These currents need to be analyzed for different type of cables to determine the minimum current required capacity for the injecting machine to make this fault detection method work reliably. Since the machine needs to be small in size for portability reason, this investigation is essential and needs to be carried out precisely.

A short pulse of energy transmitted of through a cable will be reflected from the fault location or open circuit having a shift of the waveform and containing random noise. Detection of a known wave form from the noisy signal is a challenging task. Thorough investigation is required to find the correct autocorrelation approach to correlate the noisy signals with the transmitted signal. Moreover, it is important to study the effect of non-fundamental frequency components of the dc pulse on different types of healthy cables and their contribution to noisy signals.

The design and building of the high voltage pulsar involves component selection and their operational analysis such as capacitive storage, electrical and magnetic energy storage, switch types, circuit design, and measurement test. Similarly, receiver design requires the investigation of the followings:

- Receiver sensitivity i.e. minimum required input signal $\left(\mathrm{S}_{\min }\right)$

- Transmitter and receiver integration

- Circuit complexity, protection and performance

- Misalignment tolerance i.e. acceptable influence of machine capability

- Package architecture and design

- Assembly challenges

- Safety considerations

These are the basic considerations for building up a lightweight fault locating equipment to be used by field the technician.

\section{Simulation}

Bridge methods used for locating faults in underground cables are based on modified Wheatstone circuit where direct current is used to measure the resistance in order to calculate distance of the fault in percentage of the total line length. Murray and Glaser bridges use the use similar principles for calculating the distance of the fault. Brief descriptions of these bridges are given as follows.

A Murray bridge loop for cable fault location. Besides the faulty conductor, one healthy conductor is required as connected to terminal T1 in the measuring circuit. External loop wires in the circuit, connecting the resistances at the front and the conductors at the cable end, should have close to zero resistance. Fault distances can be calculated from the resistor values using the conductor resistance per unit length of cable. 


\section{International Journal of Science and Research (IJSR) \\ ISSN (Online): 2319-7064}

Index Copernicus Value (2013): 6.14 | Impact Factor (2015): 6.391

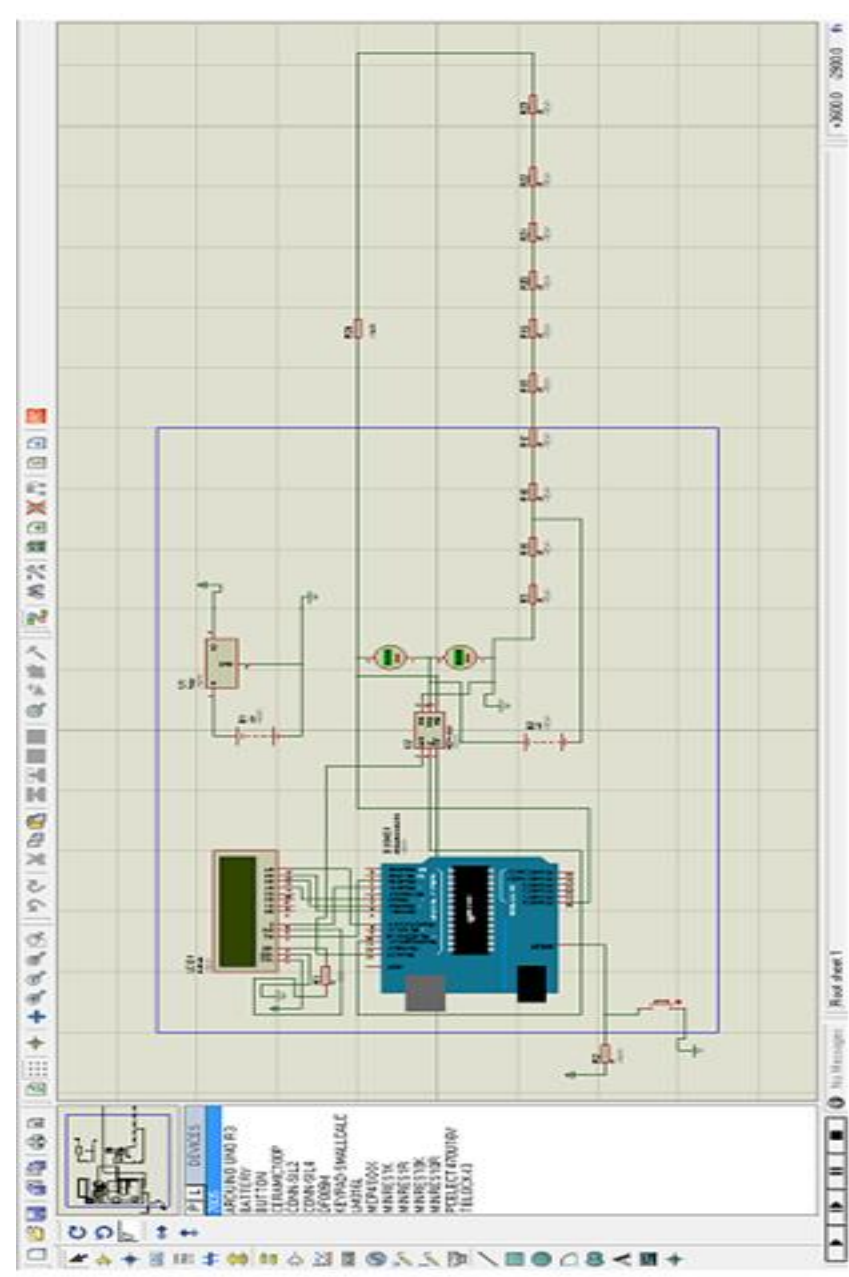

Figure: Schematic of arrangement for fault detection

\section{Simulation Results}

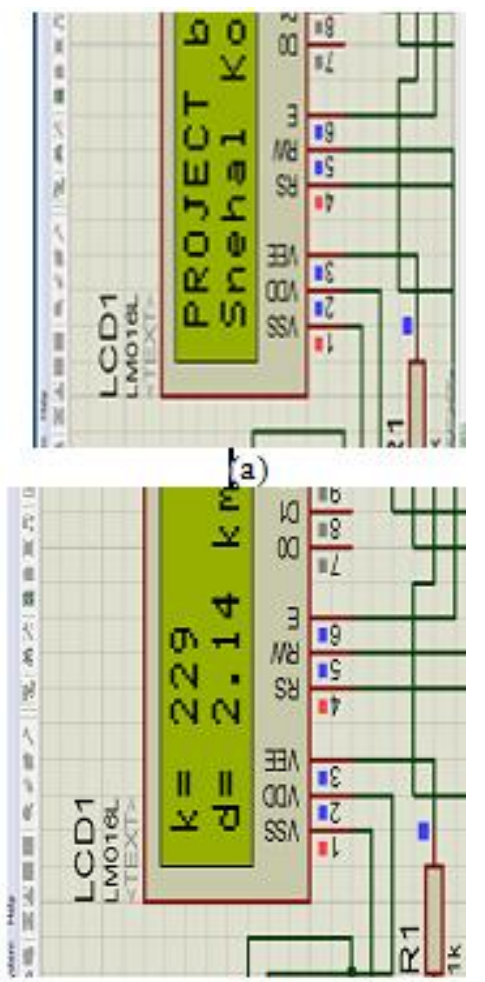

(b)

Table 1: Comparison between Actual and Calculated distance of fault in Murray Loop Test Method

\begin{tabular}{|c|c|c|}
\hline Sr.NO. & $\begin{array}{c}\text { Actual distance } \\
\text { to fault in } \mathbf{( k m})\end{array}$ & $\begin{array}{c}\text { Calculated distance to } \\
\text { fault in }(\mathbf{k m})\end{array}$ \\
\hline 1. & 1.0 & 1.05 \\
\hline 2. & 2.0 & 2.14 \\
\hline 3. & 3.0 & 3.23 \\
\hline 4. & 5.0 & 5.34 \\
\hline 5. & 8.0 & 8.46 \\
\hline
\end{tabular}

\section{Conclusion}

The simulation results show that the proposed method responds very well insensitive to fault type, fault Distance and system configuration. For the problem under consideration of simulation has been successfully applied. Therefore different types of fault analysis and fault location can be done with ease. Applied simulation methods are practically possible in field. Development for a wide range of cable length will be made in the further work in terms of safety and compact size for field measurements.

A series of tests were conducted at actual cable line sites and at the company's training facility to evaluate the locational precision of the prototype Murray loop bridges. - The potentiometer type indicated good results. Locational errors stayed around $+0.8 \%$ even under superimposition of a $230 \mathrm{~V}$ induced voltage. - The relay switching resistor type provided a similar level of locational precision compared to the potentiometer either with or without induced voltage. It could be confirmed that both prototypes have comparable performance.

\section{References}

[1] Md. Fakhrul Islam, Amanullah M T Oo, Salahuddin. A. Azad1, "Locating Underground Cable Faults: A Review and Guideline for New Development", Power Engineering Group, Faculty of sciences, Engineering and Health Central Queensland University North Rockhampton QLD 4702 Australia

[2] ShwetaGajbhiye and S.P.Karmore "Cable Fault Monitoring and Indication: A Review", International Journal of computer science and Network, Volume 2,Aug2013 ISSN(Online):2277-5420

[3] R. H. Salim, et al., "Extended fault-location formulation for power distribution systems," IEEE Transactions on Power Delivery, vol. 24, pp. 508-516, 2009.

[4] HeenaSharma,M.T.Deshpande,RahulPande "Different types of fault Analysis and Techniques of Fault Location Using PSCAD",Internation Journal of Emerging Technology and Advanced Engineering(2250-2459)May2013

[5] M. Komoda, et al., "Development of a current detection type cable fault locator," Power Delivery, IEEE Transactions on, vol. 6, pp. 541-545, 1991.

[6] C. E. T. G. Parker. (2012, 23 January). Cable and Cable Fault Locating - Part 2. Available: http://www.tracerelectronicsllc.com/resources/Applications/ Cable-Fault-Locating/FaultLocating-part2.pdf

[7] R. Salat and S. Osowski, "Accurate fault location in the power transmission line using support vector machine approach," Power Systems, IEEE Transactions on, vol. 19, pp. 979-986, 2004

Figure (a) and (b): Screen shots of Simulation Results 